\title{
Synergistic Effects of Iridoid Glycosides on the Survival, Development and Immune Response of a Specialist Caterpillar, Junonia coenia (Nymphalidae)
}

\author{
Lora A. Richards • Evan C. Lampert • \\ M. Deane Bowers • Craig D. Dodson • \\ Angela M. Smilanich • Lee A. Dyer
}

Received: 18 May 2012 /Revised: 10 September 2012 / Accepted: 16 September 2012 /Published online: 6 October 2012

(C) Springer Science+Business Media New York 2012

\begin{abstract}
Plants use a diverse mix of defenses against herbivores, including multiple secondary metabolites, which may affect herbivores synergistically. Chemical defenses also can affect natural enemies of herbivores via limiting herbivore populations or by affecting herbivore resistance or susceptibility to these enemies. In this study, we conducted larval feeding experiments to examine the potential synergistic effects of iridoid glycosides (IGs) found in Plantago spp. (Plantaginaceae) on the specialist buckeye caterpillar, Junonia coenia (Nymphalidae). Caterpillars were placed on
\end{abstract}

L. A. Richards $(\bowtie) \cdot A$. M. Smilanich $\cdot$ L. A. Dyer

Department of Biology, University of Nevada,

1664 N. Virginia Street,

Reno, NV 89557, USA

e-mail: lorar@unr.edu

E. C. Lampert • M. D. Bowers

Museum of Natural History and Department of Ecology and

Evolutionary Biology, University of Colorado,

UCB 334,

Boulder, CO 80309, USA

C. D. Dodson

Department of Physical and Environmental Sciences,

Colorado Mesa University,

1100 North Avenue,

Grand Junction, CO 81501, USA

C. D. Dodson

Division of Atmospheric Sciences, Desert Research Institute,

Reno, NV 89512, USA

Present Address:

E. C. Lampert

Department of Biological Sciences, Gainesville State University,

Gainesville, GA 30509, USA artificial diets containing different concentrations of single IGs (aucubin or catalpol alone) or combinations of the two IGs. Larval performance and immune response were recorded to test the hypothesis that IGs would have positive synergistic effects on buckeyes, which are specialists on IG plants. The positive synergistic effects that IGs had on buckeyes in our experiments included lower mortality, faster development, and higher total iridoid glycoside sequestration on mixed diets than on aucubin- or catalpol-only diets. Furthermore, we found negative synergistic effects of IGs on the immune response of buckeye caterpillars. These results demonstrate multiple synergistic effects of IGs and indicate a potential trade-off between larval performance and parasitoid resistance.

Keywords Chemical defense $\cdot$ Iridoid glycosides $\cdot$ Immune response $\cdot$ Lepidoptera $\cdot$ Nymphalidae $\cdot$ Sequestration . Synergy

\section{Introduction}

Quantifying and understanding the tritrophic effects of plant defenses have been the focus of many studies in evolutionary and chemical ecology. One "bottom-up" model posits that plant defenses directly limit herbivore populations, which in turn limit natural enemy populations (White, 1978; Abe and Higashi, 1991). However, plant defenses also can mediate "top-down" forces by attracting natural enemies to herbivores (Turlings and Wackers, 2004), by decreasing herbivore resistance to parasitoids (Bukovinszky et al., 2009; Lampert and Bowers, 2010), and by altering the susceptibility of herbivores to predators (Dyer, 1995; Nishida, 
2002). For example, sequestered toxins can negatively affect an herbivore's immune response by reducing its ability to encapsulate and kill parasitoid eggs, thus rendering them more vulnerable to successful attacks (Haviola et al., 2007; Smilanich et al., 2009). Sequestered plant defenses also can have detrimental effects on natural enemies by rendering herbivores toxic or deterrent to them (Barbosa et al., 1986; Gauld and Gaston, 1994; Ode, 2006; Singer et al., 2009).

Experiments that examine the effects of plant chemical defenses on herbivores and their enemies almost always examine individual compounds, despite evidence that many chemical defenses can be additive or synergistic (Hay et al., 1994; Nelson and Kursar, 1999; Stamp and Osier, 1998; Richards et al., 2010). For plant chemical defenses, synergy is defined as combined antiherbivore effects of different compounds that are greater than expected based on projected additive effects of each individual compound (Berenbaum et al., 1991; Jones, 1998; Nelson and Kursar, 1999). Synergy can occur between different classes of compounds or structurally similar compounds, and effects can include deterrence, toxicity, and improved or decreased herbivore performance (Jones, 1998; Scott et al., 2002; Dyer et al., 2003; Richards et al., 2010). A few studies have shown that plant chemical defenses can act synergistically to affect higher trophic interactions as well (e.g., Dyer et al., 2003; Richards et al., 2010). Since secondary metabolites are present in plants as simple or complex mixtures, widespread synergy may be partly responsible for chemical diversity within plants and for what some have termed "redundancy" in chemical defenses (Jones and Firn, 1991; Firn and Jones, 2003; Romeo et al., 1996; Rasmann and Agrawal, 2009; for microbes see Challis and Hopwood, 2003).

In this paper, we address the hypothesis that the combined actions of plant defensive compounds are synergistic and have a greater effect on herbivores than the additive effects of individual compounds acting alone. More specifically, we tested for potential synergistic effects of iridoid glycosides (IGs) on the specialist buckeye caterpillar, Junonia coenia Hübner (Nymphalidae). Buckeyes feed almost exclusively on plants containing IGs (Bowers, 1984), which are a group of cyclopentanoid monoterpene-derived compounds found in about 50 families of plants (Bobbitt and Segebarth, 1969; Jensen et al., 1975; Boros and Stermitz, 1990). Buckeye caterpillars sequester IGs (Bowers and Puttick, 1986; Bowers and Collinge, 1992), and this sequestration is an effective defense against predators (Bowers, 1980; Bowers and Farley, 1990; Dyer and Bowers, 1996; Theodoratus and Bowers, 1999). However, Smilanich et al. (2009) found that IG sequestration also may be responsible for a trade-off with parasitoid resistance. Buckeyes feeding on Plantago lanceolata, which contain high concentrations (1-12\% dry weight) of two IGs, catalpol and aucubin (Bowers and Stamp, 1993), had a weaker immune response than those feeding on Plantago major, which contain lower concentrations of aucubin only (0.2-1\% dry weight, Barton and Bowers, 2006). In these experiments, there were differences in both concentration and composition of IGs in diets that caused reduced immune response. Thus, the mechanisms affecting the immune response may have been due to concentration effects or synergistic effects. In this paper, we tested the hypotheses that iridoid glycosides act synergistically to positively affect caterpillar chemical defense against predators (sequestration of IGs) and negatively affect the immune response of buckeyes. In addition, we examined the synergistic effects of IGs on buckeye growth and development.

\section{Methods and Materials}

Study Species Junonia coenia Hübner (Lepidoptera: Nymphalidae), the common buckeye, is a specialist herbivore that is restricted primarily to plant genera that produce IGs (Bowers, 1984). Iridoid glycosides act as feeding and oviposition stimulants, with larvae refusing to feed on IGfree diets (Bowers, 1984; Pereyra and Bowers, 1988). Buckeyes have five instars and sequester IGs throughout the larval period, with the highest concentrations in newly molted 5th instars (Bowers and Collinge, 1992). During the 5 th instar, IG concentrations decrease (Bowers, unpublished data), with only small amounts found in the pupae and none in the adults (Bowers and Collinge, 1992). Larvae sequester high concentrations of IGs in their hemolymph (Bowers and Collinge, 1992), rendering them unpalatable to invertebrate predators (Camara, 1997; Theodoratus and Bowers, 1999). The concentration of IGs sequestered, and thus palatability, is dependent upon IGs available in the diet (Bowers and Collinge, 1992; Bowers and Stamp, 1997a).

Iridoid Glycoside Isolation Iridoid glycosides used in this experiment were extracted from bulk collections of Plantago lanceolata and Catalpa speciosa (Bignoniaceae). Aucubin was isolated from $P$. lanceolata, while catalpol was obtained from Catalpa speciosa. To obtain aucubin, $P$. lanceolata, leaves were dried, ground, and extracted overnight at room temperature with $95 \%$ methanol for $3 \mathrm{~d}$. Crude extracts were filtered, dried, and partitioned between water and diethyl ether. The ether layer was discarded, and the aqueous layer evaporated to dryness. The aqueous layer of P. lanceolata extracts contain both aucubin and catalpol, which are difficult to separate from each other. Therefore, the epoxide of catalpol was converted to a diol, making the compound more polar and easily separated from aucubin by flash chromatography. To achieve this $1 \mathrm{~g}$ of aqueous extract was dissolved in $80 \mathrm{ml}$ of $1 \mathrm{M} \mathrm{NaOH}$, heated to boiling, 
cooled, neutralized to pH 7.0 with $6 \mathrm{M} \mathrm{HCL}$, and dried. The solid was then triturated with methanol extensively, the methanol dried, and the residue flash chromatographed on silica gel in $4: 1 \mathrm{CHCl}_{3}: \mathrm{MeOH}$ to yield pure aucubin.

For catalpol, air dried leaves of $C$. speciosa were ground and extracted with $95 \%$ methanol for $96 \mathrm{hr}$ with periodic stirring. The methanol was evaporated, and the residue partitioned between water and diethyl ether. The ether layer was discarded, and the water layer partitioned against $n-$ butanol. The n-butanol layer was reduced in volume and then purified using flash chromatography on silica gel in 6:1 $\mathrm{CHCl}_{3}: \mathrm{MeOH}$ to obtain catalposide. The catalposide was then hydrolyzed to obtain catalpol by using an ion-exchange resin procedure outlined below (Bobbitt and Segebarth, 1969). Amberlite IRA-400 (OH) resin was washed, filtered, and rinsed $\mathrm{x} 3$ with DI water. Three grams of catalposide were dissolved in $190 \mathrm{ml}$ of DI water and stirred at $85{ }^{\circ} \mathrm{C}$ with $200 \mathrm{ml}$ of washed resin for $3 \mathrm{hr}$. The mixture was filtered, and the filtrate was washed with diethyl ether and evaporated to dryness. The used resin was then boiled in DI water for $3 \mathrm{hr}$ to release more catalpol. This mixture also was filtered, and the filtrate was washed with diethyl ether and evaporated to dryness. Combined solids were flash chromatographed on silica gel in $4: 1 \mathrm{CHCl}_{3}: \mathrm{MeOH}$ to produce pure catalpol.

Experiment We tested for synergistic effects of iridoid glycosides on buckeye caterpillars using artificial diets into which known amounts of aucubin, catalpol, or a combination were added. The base diet consisted of $11.2 \mathrm{~g}$ powdered Tobacco budworm diet (Bio-Serv, Frenchtown, NJ, USA, product \# F9781B) mixed with $66.5 \mathrm{ml}$ distilled water, $1.92 \mathrm{~g}$ agar, $380 \mu \mathrm{KOH}$, and $0.16 \mathrm{~g}$ tetracycline. For all experimental diet treatments, a single compound or a combination of compounds was dissolved in distilled water and added to the base diet. Single compound diets of aucubin and catalpol individually were $0.5,3,6$, and $12 \%$ dry weight, and the mixture diets were equal parts of each compound to make the same total IG dry weight concentrations. There were thus a total of 12 different diets. Buckeye caterpillars feed very little on diets without IGs (Bowers, 1984), thus we did not include a control diet without IG added.

Fifty newly hatched caterpillars from a buckeye laboratory colony maintained at the University of Colorado were placed on each of the 12 diet treatments. A soft paintbrush was used to transfer larvae in groups of 10 to $0.5 \mathrm{~g}$ blocks of fresh diet. Larvae were reared in a growth chamber set at $25{ }^{\circ} \mathrm{C}$ and 16:8 L:D, and checked daily to record molting and mortality. Upon molting to the 3rd instar, larvae were weighed and moved to individual plastic cups $(44 \mathrm{ml})$ until 20 larvae per treatment were separated. Growth rates for each of these larvae were calculated as the dry weight gained (mg/day) between the beginning of the 3rd instar and the beginning of 5 th instar. Furthermore, when each larva molted to the 4th (penultimate) instar, we measured food consumption over a $48 \mathrm{hr}$ period by estimating the dry weight of the diet at the beginning of the period using a DW: FW conversion factor and weighing the oven-dried diet remnants following $48 \mathrm{hr}$.

Upon reaching the 5th instar, five larvae from each treatment were randomly selected for immunocompetence experiments. When parasitoids oviposit into caterpillars, an immune response is launched in which hemocytes encapsulate the egg to asphyxiate it, or the egg is killed cytotoxically through the process of melanization (Strand, 2008). We measured the immune response using a method described by Smilanich et al. (2009). Briefly, we injected larvae with an average of four Congo red-dyed chromatography beads (DEAE Sephadex-A25, Sigma-Aldrich, St. Louis, MO, USA, product\# A25120, 40-125 $\mu \mathrm{m}$ diam) to mimic parasitoid eggs. The injected larvae continued to feed on experimental diets for $24 \mathrm{hr}$ and then were freeze killed and dissected in 5-6 $\mathrm{ml}$ methanol to retrieve the beads. Larvae were dissected in methanol to prevent break-down of IGs. Larval carcasses and associated methanol were kept and frozen for IG extraction and quantification.

The retrieved beads and a separate set of un-injected control beads were photographed under 60x magnification using an Axiocam Vision LE digital camera connected to a Leica dissecting microscope. The photos then were analyzed using Adobe Photoshop (version \#6.0, Adobe Systems Inc., San Jose, CA, USA). The red value (r-value) was measured for each bead. $\mathrm{R}$-value is an indicator of the red saturation and ranges from 0 to 255 , with 255 indicating full saturation and 0 indicating no red present. The r-values of the beads were averaged for each larva and transformed into a percent melanization $(100 *(1$-(average $\mathrm{r}$-value/control r-value) $)$ (Smilanich et al., 2009).

Chemical Analysis Larvae that were not injected with beads were allowed to pupate, then immediately prepared for IG analysis, along with the carcasses and methanol from the injected larvae. Iridoid glycosides were quantified using gas chromatography (GC) (Bowers and Stamp, 1997b; Bowers, 2003). Larvae and pupae were macerated with sand in $95 \%$ methanol, and then extracted for $48 \mathrm{hr}$. Solid material was filtered out, and the methanol was evaporated. The internal standard, phenyl- $\beta$-D-glucopyranoside (PBG) at a concentration of $0.500 \mathrm{mg} / \mathrm{ml}$, was added to each sample. The sample then was partitioned with water and diethyl ether to remove hydrophobic compounds. The ether layer was removed and the water evaporated. This residue was suspended in $1.0 \mathrm{ml}$ methanol, and a $100 \mu \mathrm{l}$ aliquot removed for analysis. Methanol was evaporated, and the remaining residue was derivatized using Tri-Syl-Z (Pierce Chemical 
Company, Rockford, IL, USA) in pyridine before injection into the GC.

Frass of 5 larvae from each treatment was collected from 4th instars. All frass egested during the 4th instar was combined for each larva, weighed, and oven-dried $48 \mathrm{hr}$ at $50{ }^{\circ} \mathrm{C}$. Oven-dried frass was ground into powder using a coffee grinder, then prepared for IG extraction using the same methods as for larvae and pupae.

For IG quantification, we used an Agilent 7890A gas chromatograph equipped with a DB-1 column $(30 \mathrm{~m}$, $0.320 \mathrm{~mm}, 0.25 \mu \mathrm{m}$ particle size), using flame-ionization detection. The temperature program used an initial temperature $200{ }^{\circ} \mathrm{C}$ held for $1 \mathrm{~min}$, followed by a $3 \mathrm{~min}$ increase to $260{ }^{\circ} \mathrm{C}$, which was held for $8 \mathrm{~min}$, followed by a $3 \mathrm{~min}$ increase to a final temperature of $320^{\circ} \mathrm{C}$, held for $10 \mathrm{~min}$. Amounts of aucubin and catalpol were quantified using ChemStation B-03-01 software.

Analysis We examined the following response variables: survival, pupal mass, development rate (1/days to pupation), growth rate (mg per day from newly molted 3rd instar to newly molted 5th instar), food consumption, melanization, IGs sequestered in larvae, and IGs excreted in frass. Residuals for all these variables in linear models were normally distributed. We examined all variables, except survival, using ANCOVA, with diet type (aucubin only, catalpol only, mixed) as a fixed factor and concentration as the covariate. We used a logistic regression to estimate the effects of diet and IG concentration on survival. We further examined the relationships between IG concentrations and buckeye pupal mass and immune response with path analyses (Proc CALIS, SAS Institute Inc., NC, USA). We chose simple models a priori based on previous results that demonstrated that IG concentration had both direct and indirect effects on pupal mass and immune response (melanization) (Smilanich et al., 2009). In the pupal mass model there were two indirect effect paths via developmental rate (1/days to pupation) and food consumption. There were also two indirect effect paths in the immune response model via growth rate (mg/day) and IG sequestration. Path analyses were conducted for all diets (aucubin-only, catalpol-only, and mixed diet) and models yielding goodness of fit chi-squares with $P$-values greater than 0.05 were considered a good fit to the data.

An interaction index $(\alpha)$ is usually calculated in synergy studies (Tallarida, 2000), and it is calculated as, $\alpha=$ $Z / \sum\left(f_{i} A_{i}\right)$. Where $f_{i}$ represents the fraction of a compound in the mixed diet and $A_{i}$ is the concentration of that compound necessary to achieve a certain level of response when tested individually; $Z$ is the concentration of the mixture of compounds necessary to achieve a certain level of response. Values of $\alpha$ significantly less than 1 demonstrate a synergy; methods for calculating variance for $\alpha$ have not been developed, thus, we calculated standard error on the basis of
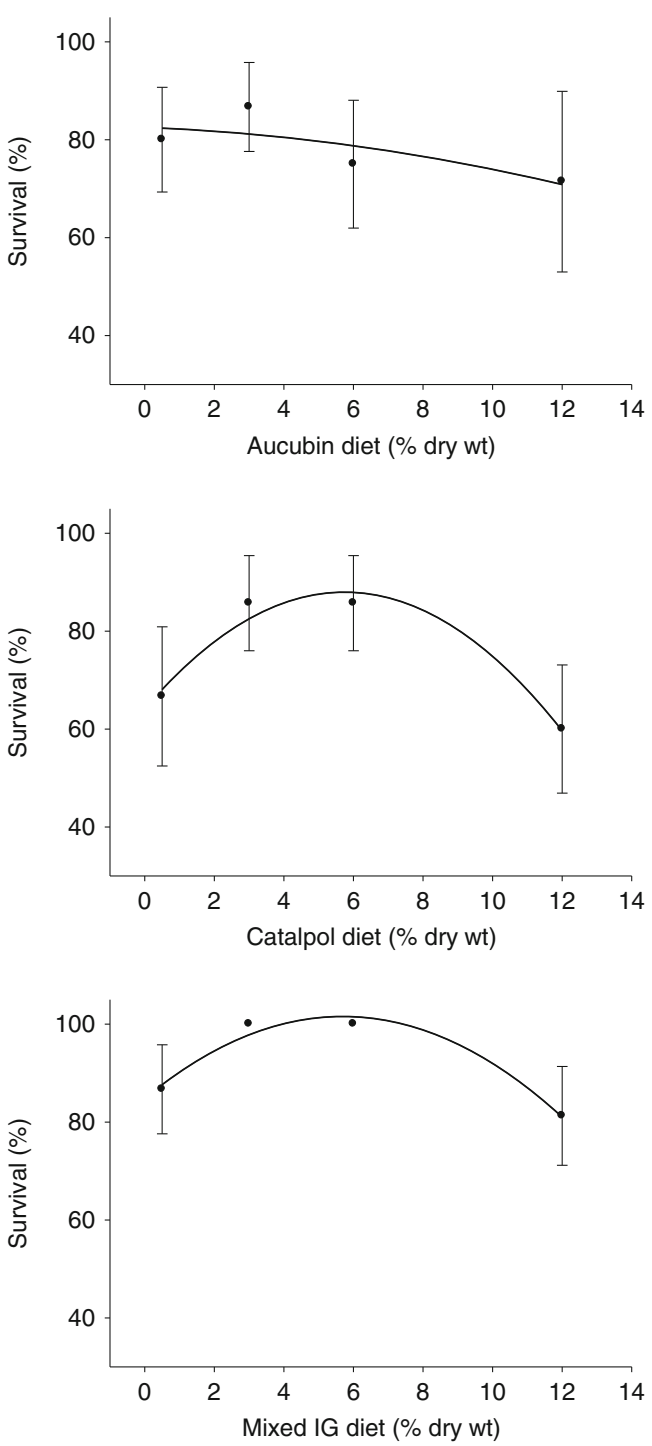

Fig. 1 Survival of buckeye larvae feeding on IG diets (mean $\pm \mathrm{SE}$ ). The $3 \%$ and $6 \%$ mixed diet graph does not have error bars due to all larvae surviving

bootstrapping from the original data $(10,000$ bootstrap resamples produced 50 new linear models from which standard errors were estimated). The interaction index often is calculated in pharmaceutical and pesticide studies where individual toxins cause strong negative effects that increase linearly with concentration. Thus, to calculate this value, individual toxins must have significant effects on the herbivore response variables when analyzed separately. We modeled the effects of concentrations of individual toxins from individual diets on the response variables, and we used parameter estimates from significant models to calculate the interaction index. If there is no relationship between concentrations of compounds in individual diets and the response variables, then any associations between concentrations in mixed diets and the response variables indicate synergy, since additive relationships cannot exist in such instances. 


\section{Results}

There were positive synergistic effects of IGs on buckeye survival and development. Overall, buckeyes had higher survival on the mixed diet $(91.6 \% \pm 3.6)$ than on aucubin $(79.6 \% \pm 5.8)$ or catalpol $(75.9 \% \pm 5.9)$ diets alone (Fig. 1). A logistic regression on survival, with diet and concentration as predictor variables, found no main effect of concentration or diet, due to high variation in survival on aucubinonly diets and a peak in survival on intermediate IG diet concentrations (Fig. 1). However, between diet comparisons found that survival was significantly greater on the mixed diet than the catalpol-only diet (Logistic regression, $\chi^{2}=$ 8.81, $d f=3, P<0.05, \beta=1.24 \pm 0.57$, Wald $=4.73, d f=1, P>$ 0.05 , Fig. 1) and was not significantly different between aucubin-only and catalpol-only diets.

Buckeye developmental rates were highest on a mixed diet at an intermediate concentration (ANCOVA, diet effect $F_{2,135}=8.77, P<0.001$; there was no significant effect of concentration; Fig. 2). The quadratic curve that fits the data indicates that buckeyes not only reach higher maximum developmental rates on a mixed diet, but that this maximum is achieved at higher concentrations than on single IG diets (Fig. 2, aucubin diet curve fit $y=0.024+0.0008 x-0.0000$ $6 x^{2}, R^{2}=0.17$, vertex $=(5.82,0.0266), F_{2,38}=3.65, P=0.04$; catalpol diet curve fit $y=0.023+0.0011 x-0.00009 x^{2}$, $R^{2}=0.34$, vertex $=(6.12,0.0261), F_{2,40}=9.64, P<0.001$; mixed diet curve fit $y=0.023+0.0017 x-0.0001 x^{2}, R^{2}=$
0.48, vertex $\left.=(7.50,0.0293), F_{2,55}=24.00, P<0.001\right)$. From these models, we calculated an interaction index $(\alpha)$ of 0.42 (as described in the methods, values less than 1 indicate synergy) on the effective dose to increase development rate by $10 \%(\mathrm{ED}+10$, from the average development time from all 0.5 diets). The bootstrap mean and standard error of the interaction index were $0.58,0.11$ SEM, indicating that $\alpha$ was significantly nonnegative and lower than 1.0 - the cutoff value for synergy. An ANCOVA revealed that pupal mass covaried with IG concentration $\left(F_{1,135}=6.36, P<0.05\right.$, there was no significant effect of diet). However, path analysis supported the hypothesis that there are positive synergistic effects on development that subsequently cause positive synergistic effects on pupal mass (Fig. 2). On single IG diets, there was an indirect negative effect on pupal mass (aucubin diet, Path model $\chi^{2}=0.17, d f=1$, $P=0.68$; catalpol diet, Path model, $\chi^{2}=0.19, d f=1, P=$ 0.66). In comparison, on a mixed diet there was a positive indirect effect (Path Model $\chi^{2}=0.62, d f=1$, $P=0.43$, Fig. 2).

There were both concentration and diet effects on total IGs sequestered in larvae and pupae, and excreted in frass (Fig. 3). By the early 5 th instar, larvae had sequestered more IG on mixed diets than on single diets. For example, on $3 \%$ mixed diets, larvae sequestered $146 \%$ and $83 \%$ more total IGs than on catalpol or aucubin-only, respectively (total IG sequestered concentration $F_{1,52}=10.95, P<0.01$, diet effect $F_{2,52}=6.40, P<0.01$; aucubin sequestered, concentration
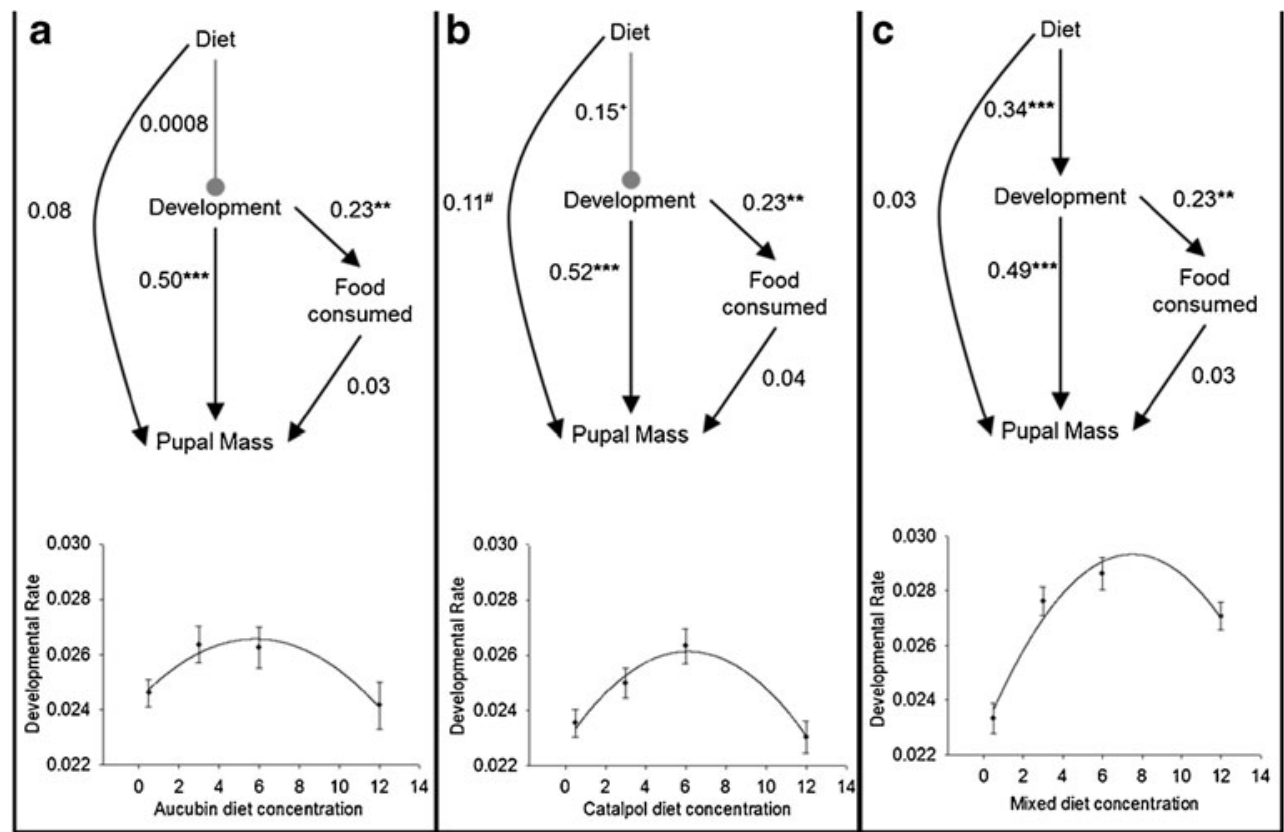

Fig. 2 Effects of iridoid glycosides on the pupal mass and development of buckeye larvae. Positive effects between variables are indicated by a black arrow, and negative effects are indicated by a grey bullet. Relationship strengths are indicated by the standardized path coefficient. Development is measured as days ${ }^{-1}$ to pupation. Scatterplots and second order curves for developmental rate and diet concentration are inset. a Direct and indirect effects of aucubin diets on buckeyes. b Effects of catalpol diets on buckeyes. $\mathbf{c}$ Effects of mixed IG diets on buckeyes. The significance of the path coefficients are indicated as the following: ***<0.001, **<0.01, *<0.05, $+<0.1, \#<0.2$ 
$F_{1,34}=17.32, P<0.01$, no effect of diet; catalpol sequestered, no effect of concentration and diet). Larvae on mixed diets reached a maximum amount sequestered on the $3 \%$ diet, whereas the maximum was reached at $6 \%$ on the aucubin diet. On the catalpol diet, a lower maximum was reached on $6 \%$ diet. These differences between mixed and single diets were also found in the frass. Fourth instars excrete more IGs on mixed diets than on single diets (Fig. 3a, concentration $F_{1,57}=33.08, P<0.001$, diet effect $F_{2,57}=17.79, P<0.001$.). The pupae from mixed diets had higher total IG content, although when comparing concentrations of individual IGs (aucubin and catalpol) within the mixed diet, we found that they were lower than concentrations found in pupae from single IGs diets (total IG sequestered, concentration $F_{1,134}=8.10, P<$ 0.005 , diet effect $F_{2,134}=2.98, P=0.05$; Aucubin sequestered; concentration covariate $F_{1,101}=4.78, P<0.05$, diet effect $F_{1}$, ${ }_{101}=11.67, P<0.001$; Catalpol sequestered, concentration $F_{1,96}=5.53, P<0.05$, diet effect $F_{1,96}=9.16, P<0.01$ ).

There was no effect of concentration or diet on melanization when analyzing the data in isolation (ANCOVA, $P>$ 0.05 ). However, when we included multiple pathways by which IG concentrations can affect melanization, we found direct negative synergistic effects of diet on the immune response (Fig. 4. aucubin path model, $\chi^{2}=0.33, d f=1, P=$ 0.56 , catalpol path model, $\chi^{2}=0.80, d f=1, P=0.37$, aucubin in mixed diet path model, $\chi^{2}=0.16, d f=1, P=0.32$, catalpol in mixed diet path model, $\chi^{2}=0.05, d f=1, P=0.81$ ). It is clear from path analysis (Fig. 4) that direct negative and indirect positive pathways cancel each other, which explains why there were no significant effects in the ANCOVA model.

Similarly, there was no concentration or diet effect on catalpol sequestration (Fig. 3). However, in the path model, we found that catalpol sequestration was affected more by diet concentration in a mixed diet than in the catalpol-only diet (Fig. 4). Although there was a concentration effect on growth rate (ANCOVA $F_{1,135}=6.80, P<0.05$ ), there was no effect of diet, which also was supported by the path analysis.

The summed coefficients of the direct and indirect effects on melanization of aucubin were -0.04 for the aucubin diet and -0.20 for aucubin in the mixed diet, in comparison to 0.19 on the catalpol diet and -0.29 for catalpol in the mixed diet. The larger absolute summed coefficients in the catalpol models indicate that catalpol had a greater effect on melanization than aucubin. In addition, there was an overall positive effect on melanization on a catalpol-only diet and a negative effect of catalpol on a mixed diet. This sign shift demonstrates the negative synergistic effect on melanization.

\section{Discussion}

We found multiple synergistic effects of IGs on buckeyes. On mixed diets, larvae developed faster, had higher
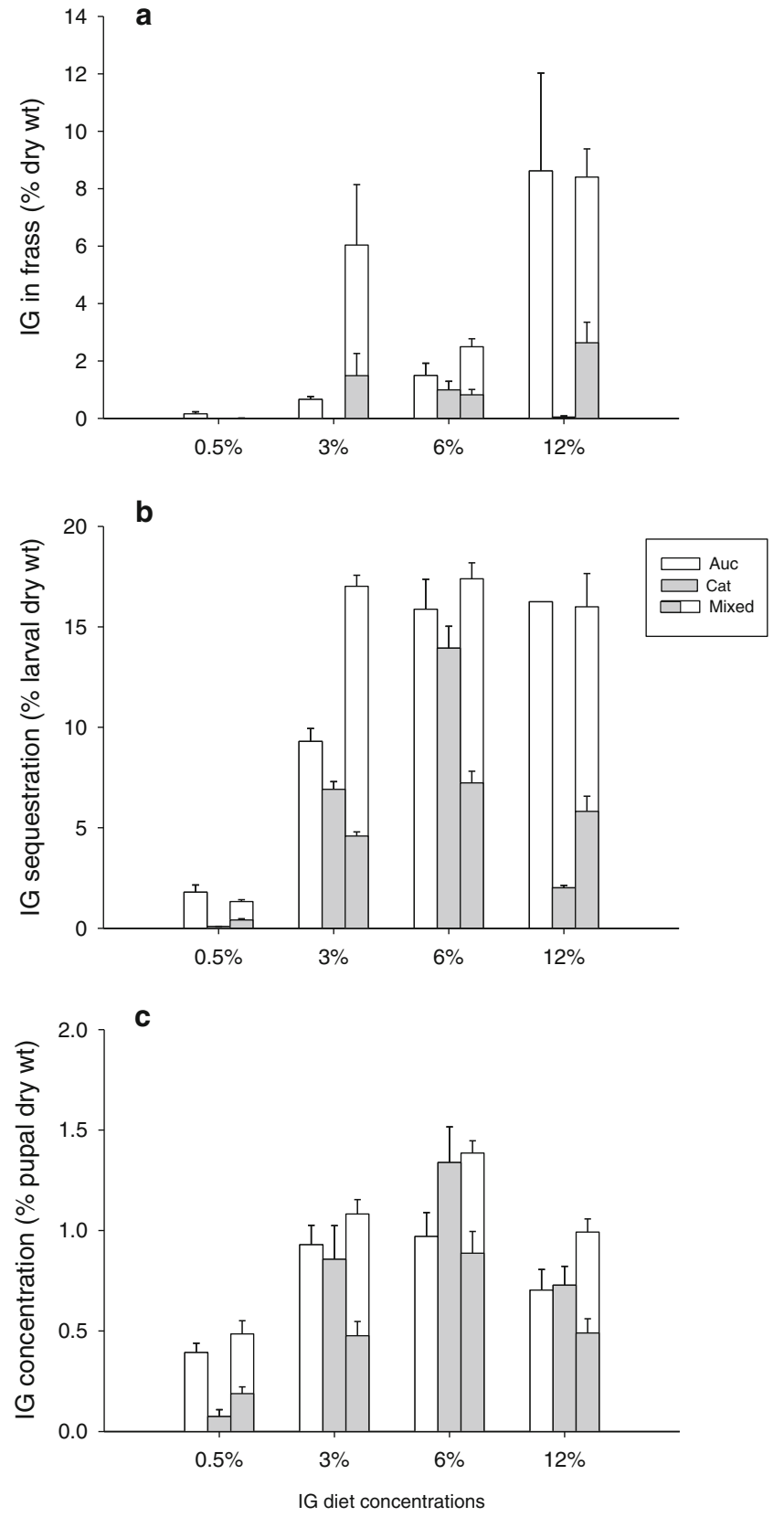

Fig. 3 Iridoids excreted and sequestered (mean \pm SE) by buckeyes on single and mixed diets. a Total IG in frass from the entire 4th instars significantly varied with concentration and was significantly different between diets. b Total IG sequestered by larvae at the time they start the 5th instar significantly varied with concentration and was significantly different between diets. c Total IG in pupae significantly varied with concentration and was significantly different between diets

survival, sequestered more IGs, and had a reduced immune response compared to single IGs diets. These synergistic effects have interesting tritrophic implications. Despite the fact that sequestration by buckeyes results in a strong defensive benefit against a variety of predators (Bowers, 1980; Bowers and Farley, 1990; Dyer and Bowers, 1996; Camara, 1997; Theodoratus and Bowers, 1999; Rayor and Munson, 
Fig. 4 Effects of iridoid glycosides on the immune response of buckeye larvae. The top diagrams show the relationships for larvae on single IG treatments. The bottom diagrams show the relationships for larvae on the mixed amide treatment. The significance of the path coefficients are indicated as the following: $* * *<0.001, * *$ $<0.01, *<0.05,+<0.1, \#<0.2$
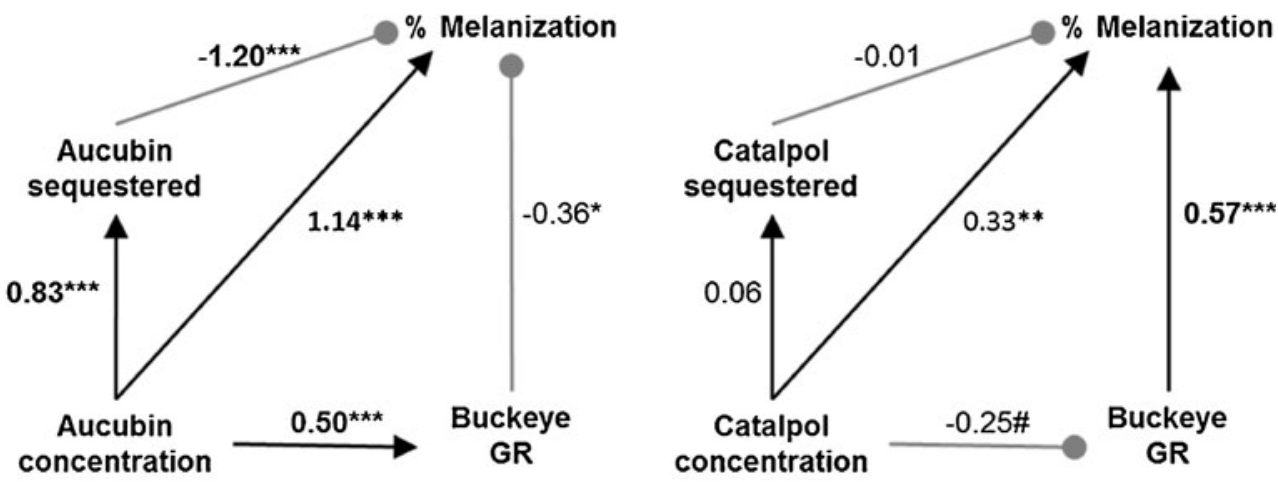

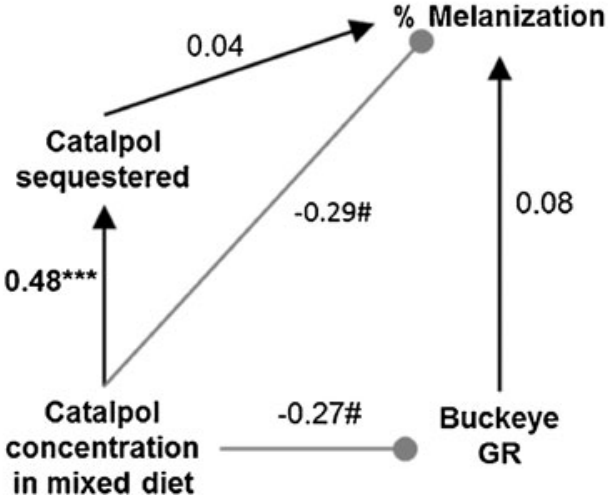

2002), our results are consistent with Smilanich et al. (2009), showing a negative effect of plant chemical defenses on the caterpillar immune response. As with the Smilanich et al. (2009) experiments in which buckeyes were fed Plantago major, which has low concentrations of aucubin only, we found that the IG variation in aucubin-only diets had little effects on melanization. In comparison, larvae feeding on a mixed diet, similar to $P$. lanceolata with both aucubin and catalpol, incurred negative effects on melanization. Since mixed diets were all at the same concentrations, our results support the idea that the decreased immune response in buckeye feeding on $P$. lanceolata likely is due to the combination of synergistic effects and concentration effects.

For specialist parasitoids, buckeyes may provide enemyfree space for parasitoid larvae, which benefit from the reduced predation of their host. In other species of caterpillars that specialize on IGs (Melitaea cinxia, Nymphalidae and Ceratomia catalpa, Sphingidae), specialist parasitoids are unaffected by larval IG content, and thus may find a safe-haven inside the chemically defended host (Harvey et al., 2005; Lampert et al., 2010; Reudler et al., 2011). This apparent trade-off between predator protection and parasitoid resistance does not necessarily apply to all parasitoids or all aspects of buckeye caterpillar biology. For example, there were positive synergistic effects on buckeye development and survival. By developing faster on a mixed diet, buckeyes may reduce the window during which they are vulnerable to parasitoid attack (Haggstrom and Larsson, 1995; Turlings and Benrey, 1998; Fordyce and Shapiro, 2003). In addition, we measured only one mechanism of parasitoid resistance, so it is not clear if other components of parasitoid resistance are negatively affected. In fact, high concentrations of sequestered IGs may be toxic for many non-specialist parasitoid larvae (Nishida, 2002).

Iridoid glycosides that are produced by plants are defensive, yet we found positive synergistic effects on larval performance of specialist buckeye caterpillars. Previous studies have found that plant defenses synergistically affect generalist and specialist herbivores differently (Dyer et al., 2003; Richards et al., 2010). Generalists experience more direct effects of toxicity, whereas the effects on specialists are more subtle. As with the results reported here for IGs, amides found in Piper plants synergistically weaken the immune response and increase parasitism rates of specialist caterpillars, Eois spp. (Dyer et al., 2003; Richards et al., 2010). However, unlike Eois, buckeyes performed better on relatively high IG content diets. Even on the highest concentration of mixed diet (12\%), for which there was a slightly detrimental effect, buckeyes often performed better than on the lowest concentration $(0.5 \%)$ of any mixed or single diet.

In a previous study, Camara (1997) found that on mixed diets buckeyes were the most efficient in sequestering IGs at intermediate concentrations. He quantified IG sequestration 
by using a modified index of the efficiency of conversion of ingested food (ECI) calculated as the IG in larvae divided by the IG consumed by the larvae $\left(\mathrm{ECI}_{\mathrm{IG}}\right)$. He compared $\mathrm{ECI}_{\mathrm{IG}}$ values in buckeyes feeding on artificial diets with trace, $2 \%$ and $10 \%$ P. lanceolata extracts and found that IG sequestration was most efficient on the $2 \%$ diet. In comparison, Bowers and Collinge (1992) found that IG sequestration was inefficient (low $\mathrm{ECI}_{\mathrm{IG}}$ ) on artificial diets that contained $2 \%$ aucubin or catalpol (single IG diets). While we did not measure sequestration efficiency directly, these differences in sequestration efficiencies may help explain why larvae appeared to reach a threshold of IG sequestration on a $3 \%$ concentration in mixed diet, whereas on the single diets the maximum sequestration was reached on the $6 \%$ diets.

Previous studies also have shown that buckeyes exhibit preference for ovipositing on $P$. lanceolata compared to $P$. major (Prudic et al., 2005). One interpretation of such results is that the female is choosing the high IG species that better protects her offspring from predators (Dyer and Bowers, 1996). Theodoratus and Bowers (1999) found that buckeye larvae reared on $P$. lanceolata were better defended from wolf spiders than those reared on P. major. Given the known protective role of IGs against large generalist predators, and the detrimental effects on the immune response, we can speculate that parasitoid enemies may not have played a large selective role in the adaptation of these caterpillars to their host plant chemistry. This study and previous work (Smilanich et al., 2009) support this assumption. However, our measure of the immune response was on limited parameters (encapsulation and melanization), and it is possible that other parameters may respond differently (i.e., antimicrobial peptides, lysozyme activity) (Adamo 2010). Nonetheless, the encapsulation and melanization responses are the most effective for large, eukaryotic invaders such as parasitoids (Carton et al., 2008).

In summary, we found multiple synergistic effects of IGs on buckeyes that have important implications for their relationship with natural enemies. Aucubin and catalpol had negative synergistic effects on the larval immune response, which may make them more susceptible to parasitoid induced mortality. However, the cost of a reduced immune response may be outweighed by the positive synergistic effects on larval survival, developmental rates, and sequestration. An increase in developmental rates decreases the time that caterpillars are susceptible to attack by natural enemies, while enhanced sequestration efficacy and higher larval IG content may be more deterrent to non-parasitoid predators. This study further highlights the importance of investigating synergies between plant chemical defenses and their effects on multiple trophic levels. To date, it has been difficult to test the role of synergy in the general phytochemical diversity hypothesis proposed by Jones and Firn (1991), but results with other mixtures from distinct classes of compounds that demonstrate synergistic effects across trophic levels should continue to reveal the importance of phytochemical redundancy for plant fitness.

Acknowledgments This work was supported by Earthwatch Institute, UNR and National Science Foundation grants DEB1020509 and DEB0849361 to L.A.D., and DEB0614883 to M.D.B. For comments and discussion on the manuscript, we thank N. Pardikes, A. Glassmire, K. Fredrickson, and C. Jeffrey.

\section{References}

ABE, T. and Higashi, M. 1991. Cellulose centered perspective on terrestrial community structure. Oikos 60:127-133.

ADAMO, S. A. 2010. Why should an immune response activate the stress response? Insights from the insects (the cricket Gryllus texensis). Brain Behav. Immun. 24:194-200.

Barbosa, P., SAunders, J. A., Kemper, J., Trumbule, R., Olechno, J., and MARTINAT, P. 1986. Plant allelochemicals and insect parasitoids effects of nicotine on Cotesia-Congregata (Say) (Hymenotpera, Braconidae) and Hyposoter-Annulipes (Cresson) (Hymenotpera, Ichneumonidae). J. Chem. Ecol. 12:1319-1328.

BARTON, K. E. and Bowers, M. D. 2006. Neighbor species differentially alter resistance phenotypes in Plantago. Oecologia. 150:442-452.

Berenbaum, M., Nitao, J. K., and Zangerl, A. R. 1991. Adaptive significance of furanocoumarin diversity in Pastinaca sativa (Apicaceae). J. Chem. Ecol. 17:207-215.

BobBitT, J. M. and SEgEBARTH, D. P. 1969. Iridoid glycosides and similar substances, pp. 1-145, in W. I. Taylor and A. R. Battersby (eds.), Cyclopentanoid terpene derivatives. Academic, New York.

Boros, C. A. and Stermitz, F. R. 1990. Iridoids: An updated review. Part 1. J. Nat. Prod. 53:1055-1147.

BOWERS, M. D. 1980. Unpalatability as a defense strategy of Euphydryas phaeton (Lepidoptera: Nymphalidae). Evolution 34:586-600.

BOWERS, M. D. 1984. Iridoid glycosides and host-plant specificity in larvae of the buckeye butterfly, Junonia-Coenia (Nymphalidae). J. Chem. Ecol. 10:1567-1577.

Bowers, M. D. 2003. Hostplant suitability and defensive chemistry of the Catalpa sphinx, Ceratomia catalpae. J. Chem. Ecol. 29: 2359-2367.

Bowers, M. D. and Collinge, S. K. 1992. Fate of iridoid glycosides in different life stages of the buckeye, Junonia-Coenia (Lepidoptera, Nymphalidae). J. Chem. Ecol. 18:817-831.

Bowers, M. D. and FARLEY, S. D. 1990. The behaviour of gray jays (Perisoreus canadensis) towards palatable and unpalatable Lepidoptera. Anim. Behav. 39:699-705.

Bowers, M. D. and PutTick, G. M. 1986. Fate of ingested iridoid glycosides in lepidopteran herbivores. J. Chem. Ecol. 12:169178.

Bowers, M. D. and STAMP, N. E. 1993. Effects of plant-age, genotype, and herbivory on plantago performance and chemistry. Ecology. 74:1778-1791.

BOWERS, M. D. and STAMP, N. E. 1997a. Effect of hostplant genotype and predators on iridoid glycoside content of pupae of a specialist insect herbivore, Junonia coenia (Nymphalidae). Biochem. Syst. Ecol. 25:571-580.

Bowers, M. D. and STAMP, N. E. 1997b. Fate of host-plant iridoid glycosides in lepidopteran larvae of Nymphalidae and Arctiidae. J. Chem. Ecol. 23:2955-2965.

Bukovinszky, T., Poelman, E. H., Gols, R., Prekatsakis, G., Vet, L. E. M., HARVEY, J. A., and DiCKE, M. 2009. Consequences of constitutive and induced variation in plant nutritional quality for 
immune defence of a herbivore against parasitism. Oecologia 160:299-308.

CAmarA, M. D. 1997. Predator responses to sequestered plant toxins in buckeye caterpillars: Are tritrophic interactions locally variable? $J$. Chem. Ecol. 23:2093-2106.

Carton, Y., Poirie, M., and Nappi, A. J. 2008. Insect immune resistance to parasitoids. Insect Sci. 15:67-87.

Challis, G. L. and HopwOOD, D. A. 2003. Synergy and contingency as driving forces for the evolution of multiple secondary metabolite production by Streptomyces species. Proc. Natl. Acad. Sci. 100:14555-14561.

DYer, L. A. 1995. Tasty generalists and nasty specialists - Antipredator mechanisms in tropical lepidopteran larvae. Ecology 76: $1483-1496$.

DYER, L. A. and BOWERS, M. D. 1996. The importance of sequestered iridoid glycosides as a defense against an ant predator. J. Chem. Ecol. 22:1527-1539.

Dyer, L. A., Dodson, C. D., Stireman III, J. O., Tobler, M. A., Smilanich, A. M., Fincher, R. M., and Letourneau, D. K. 2003. Synergistic effects of three Piper amides on generalist and specialist herbivores. J. Chem. Ecol. 29:24992514.

FIRN, R. D. and JONES, C. G. 2003. Natural products - A simple model to explain chemical diversity. Nat. Prod. Rep. 20:382-391.

FordycE, J. A. and SHAPIRO, A. M. 2003. Another perspective on the slow-growth/high-mortality hypothesis: Chilling effects on swallowtail larvae. Ecology 84:263-268.

GAULD, I. D. and GASTON, K. J. 1994. The taste of enemy-free space: Parasitoids and nasty hosts, pp. 279-299, in B. A. Hawkins and W. Sheehan (eds.), Parasitoid Community Ecology. Oxford University Press, New York.

HAGGSTROM, H. and LARSSON, S. 1995. Slow larval growth on a suboptimal willow results in high predation mortality in the leaf beetle galerucella-lineola. Oecologia 104:308-315.

Harvey, J. A., VAn NouhuYs, S., and Biere, A. 2005. Effects of quantitative variation in allelochemicals in Plantago lanceolata on development of a generalist and a specialist herbivore and their endoparasitoids. J. Chem. Ecol. 31:287-302.

Haviola, S., Kapari, L., Ossipov, V., Rantala, M. J., RuUhola, T., and HAUKIOJA, E. 2007. Foliar phenolics are differently associated with Epirrita autumnata growth and immunocompetence. $J$. Chem. Ecol. 33:1013-1023.

HaY, M. E., Kappel, Q. E., and Fenical, W. 1994. Synergisms in plant defenses against herbivores - ineractions of chemistry, calcification, and platy-quality. Ecology 75:1714-1726.

Jensen, S. R., NiELSEN, B. J., and DAHLGREN, R. 1975. Iridoid compounds, their occurrence and systematic importance in the angiosperms. Bot. Notiser 128:148-180.

JONES, C. G. and FIRN, R. D. 1991. On the evolution of plant secondary chemical diversity. Phil. Trans. R. Soc. B 333:273-280.

Jones, D. G. 1998. Piperonyl Butoxide: The Insect Synergist. Academic Press, London.

LAMPERT, E. C. and Bowers, M. D. 2010. Host plant species affects the quality of the generalist Trichoplusia $n i$ as a host for the polyembryonic parasitoid Copidosoma floridanum. Entomol. Exp. Appl. 134:287-295.

LAMPERT, E. C., Dyer, L. A., and Bowers, M. D. 2010. Caterpillar chemical defense and parasitoid success: Cotesia congregata parasitism of Ceratomia catalpae. J. Chem. Ecol. 36:992998.
Nelson, A. C. and Kursar, T. A. 1999. Interactions among plant defense compounds: A method for analysis. Chemoecology 9:81-92.

NisHIDA, R. 2002. Sequestration of defensive substances from plants by Lepidoptera. Annu. Rev. Entomol. 47:57-92.

ODE, P. J. 2006. Plant chemistry and natural enemy fitness: Effects on herbivore and natural enemy interactions. Annu. Rev. Entomol. $51: 163-185$

PEREYRA, P. C. and Bowers, M. D. 1988. Iridoid glycosides as oviposition stimulants for the buckeye butterfly, Junonia coenia (Nymphalidae). J. Chem. Ecol. 14:917-928.

Prudic, K. L., Oliver, J. C., and Bowers, M. D. 2005. Soil nutrient effects on oviposition preference, larval performance, and chemical defense of a specialist insect herbivore. Oecologia 143:578-587.

Rasmann, S. and Agrawal, A. A. 2009. Plant defense against herbivory: Progress in identifying synergism, redundancy, and antagonism between resistance traits. Curr. Opin. Plant Biol. 12:473-478.

RAYOR, L. S. and MunSON, S. 2002. Larval feeding experience influences adult predator acceptance of chemically defended prey. Entomol. Exp. Appl. 104:193-201.

Reudler, J. H., Biere, A., Harvey, J. A., and van Nouhuys, S. 2011. Differential performance of a specialist and two generalist herbivores and their parasitoids on Plantago lanceolata. J. Chem. Ecol. 37:765-778.

Richards, L. A., Dyer, L. A., SMilanich, A. M., and Dodson, C. D. 2010. Synergistic effects of amides from two piper species on generalist and specialist herbivores. J. Chem. Ecol. 36:1105-1113.

RomeO, J. T., SAunders, J. A., and Barbosa, P. 1996. Phytochemical Diversity and Redundancy in Ecological Interactions. Plenum, New York.

Scott, I. M., Puniani, E., Durst, T., PhelPs, D., Merali, S., AssabGui, R. A., SÁnchez-VIndas, P., Poveda, L., Philogéne, B. J. R., and ARNASON, J. T. 2002. Insecticidal activity of Piper tuberculatum Jacq. extracts: Synergistic interaction of piperamides. Agr. For. Entomol. 4:137-144.

Singer, M. S., Mace, K. C., and Bernays, E. A. 2009. Selfmedication as adaptive plasticity: Increased ingestion of plant toxins by parasitized caterpillars. PLoS One 4.

Smilanich, A. M., Dyer, L. A., Chambers, J. Q., and Bowers, M. D. 2009. Immunological cost of chemical defence and the evolution of herbivore diet breadth. Ecol. Lett. 12:612-621.

STAMP, N. E. and OSIER, T. L. 1998. Response of five insect herbivores to multiple allelochemicals under fluctuating temperatures. Entomol. Exp. Appl. 88:81-96.

STRand, M. R. 2008. The insect cellular immune response. Insect Sci. 15:1-14.

TALlaridA, R. J. 2000. Drug synergism and dose-effect data analysis. Chapman and Hall/CRC, Boca Raton, FL.

THEODORATUS, D. H. and BowERS, M. D. 1999. Effects of sequestered iridoid glycosides on prey choice of the prairie wolf spider, Lycosa carolinensis. J. Chem. Ecol. 25:283-295.

TurLings, T. C. J. and BENREY, B. 1998. Effects of plant metabolites on the behavior and development of parasitic wasps. Ecoscience 5:321-333.

TURLINGS, T. C. J. and WACKERS, F. 2004. Recruitment of predators and parasitoids by herbivore-injured plants, pp. 21-75, in R. T. Carde and J. G. Millar (eds.), Advances in insect chemical ecology. Cambridge University Press, UK.

White, T. C. R. 1978. Importance of a relative shortage of food in animal ecology. Oecologia 33:71-86. 